\title{
Parametric Study of Variable Emissivity Radiator Surfaces
}

\author{
Lisa M. Grob ${ }^{1}$ and Theodore D. Swanson ${ }^{2}$ \\ 'Swales Aerospace, Beltsville MD 20705 \\ 'NASA Goddard Space Flight Center, Code 545, Greenbelt MD 20771
}

\begin{abstract}
The goal of spacecraft thermal design is to accommodate a high function satellite in a low weight and real estate package. The extreme environments that the satellite is exposed during its orbit are handled using passive and active control techniques. Heritage passive heat rejection designs are sized for the hot conditions and augmented for the cold end with heaters. The active heat rejection designs to date are heavy, expensive and/or complex. Incorporating an active radiator into the design that is lighter, cheaper and more simplistic will allow designers to meet the previously stated goal of thermal spacecraft design. Varying the radiator's surface properties without changing the radiating area (as with VCHP), or changing the radiators' views (traditional louvers) is the objective of the variable emissivity (vary-e) radiator technologies. A parametric evaluation of the thermal performance of three such technologies is documented in this paper. Comparisons of the Micro-Electromechanical Systems (MEMS), Electrochromics, and Electrophoretics radiators to conventional radiators, both passive and active are quantified herein. With some noted limitations, the vary-e radiator surfaces provide significant advantages over traditional radiators and a promising alternative design technique for future spacecraft thermal systems.
\end{abstract}

\section{INTRODUCTION}

A parametric study of vary-e radiator surfaces is documented within. The scope of the study ranges from steady state heat balance equations to modified EOS-AM Spacecraft Thermal Math Models (TMMs). Thermal performance, cost and weight of the MEMS, Electrochromics and Electrophoretics vary-e surfaces were compared with conventional satellite radiator surfaces. The significant savings in heater power, cost and weight illustrate the advantages vary-e radiator surfaces have over traditional surfaces. The savings these new technologies offer when a reduction in heat loss is required to maintain satellite temperatures can provide smaller, less complex and cheaper satellites.

Detailed descriptions of the variable emissivity radiator surfaces and EOS-AM TMMs are beyond the scope of this paper.

\section{DISCUSSION OF ANALYSIS}

An incremental study approach was used to progressively assess the thermal performance of these vary-e radiators. The orbits and environments used in all phases of the study were selected to reflect the EOS-AM spacecraft design criteria since the final analysis included a comparison with the EOS battery radiators. EOS-AM mission orbit is a sun-synchronous 10:30 descending node, $98.2^{\circ}$ inclination polar orbit at an altitude of $705 \mathrm{~km}$.

Initially, steady state calculations, using orbital average fluxes were performed on a number of radiator surfaces. The calculated heat rejection capabilities of traditional radiators were compared against the three vary-e radiator surfaces. A "baselined" Silver Teflon (AgTeflon) radiator, sized at 350 in $^{2}$ was used to 
maintain a $30^{\circ} \mathrm{C}$ panel supporting a $75 \mathrm{~W}$ "box". This design was evaluated using a Ag Teflon constant conductance heat-pipe pancl (CCHP), a Ag Teflon variable conductance heat-pipe panel (VCHP), a traditional louvered CCHP panel surfaced with $\mathrm{Ag}$ Teflon, a MEMS CCHP radiator, an Electrochromics CCHP radiator and an Electrophoretic CCHP radiator. All technologies were assessed using realistic designs based on the hot case parameters defined in Table 1. Radiator areas for traditional louvers and Electrochromics used in the comparison reflected area inefficiencies and non-optimized thermal optical properties. The actual radiator area of the traditional louvered radiator, was 425 in $^{2}$ due to area inefficiencies. Electrochromics radiator was evaluated at $400 \mathrm{in}^{2}$ because of the higher solar absorptance. Once the hot case was defined for all the surfaces, cold case calculations that included beginning of life (BOL) properties and no box dissipations, determined the heater power required to maintain the radiators at $-10^{\circ} \mathrm{C}$.

Of particular interest are the spacecraft "cold side" $(+Y)$ radiators where most of the housekeeping equipment (including the nickel hydrogen batteries) resides and the instrument deck on the nadir side $(+Z)$ for the typical LEO science mission. Both the nadir and cold side radiators experience mainly albedo and IR fluxes. The albedo is significant, however in the low beta orbits. During the year, the EOS-AM spacecraft has a beta range of $13^{\circ}$ to $32^{\circ}$. The zenith $(-Z)$ radiator was also studied since a significant portion of the EOS-AM housekeeping equipment is accommodated there. The zenith radiator experiences direct solar fluxes, which emphasizes the significance and/or limitations of the current vary-e technologies' absorptivities.

The analysis parameters and assumptions for the steady state calculations used to evaluate the different radiator technologies are found in Table 1. Figure 1 is an illustration of the radiator comparisons.

Table 1. Steady State Analysis Assumptions and Parameters

Typical sun-synchronous LEO orbit / beta range $13^{\circ}$ and $32^{\circ}$

Hot Case Models EOL environment and properties on the spacecraft cold side radiator 75 watt box mounted on the $30^{\circ} \mathrm{C}$ panel

Cold Case Models BOL environment and properties on the spacecraft cold side radiator Panel heaters maintaining a $-10^{\circ} \mathrm{C}$ panel

Radiator Surface Properties

\begin{tabular}{lll}
\hline & BOL & EOL \\
Ag Teflon & 0.08 & 0.18 \\
OSRs & 0.08 & 0.14 \\
White Paint & 0.15 & 0.40 \\
Electrochromics & 0.30 & 0.30 \\
MEMS & 0.20 & 0.20 \\
Electrophoretics & 0.20 & 0.20
\end{tabular}

Effective emissivity of closed louver is 0.090

$\alpha$ $\varepsilon$

$\begin{array}{ll}0.79 & \\ 0.80 & \\ 0.85 & \\ 0.29^{[1]} & 0.70^{[2]} \\ 0.20^{[1]} & 0.80^{[2]} \\ 0.29^{[1]} & 0.80^{[2]}\end{array}$

[1] light/closed/activated state

[2] dark/open/base state

In addition to the hot and cold case scenarios discussed above, heat rejection capability was calculated as a function of radiator area using the parameters outlined in Table 1. The resulting plots for the hot and cold cases, see Figures 2 through 9 , reveal the heat rejection capabilities from the zenith, nadir and cold side radiators for areas up to $1000 \mathrm{in}^{2}$. It is evident from Figures 2 and 4 that the Electrochromics and MEMS radiators will not run as cold as $-10^{\circ} \mathrm{C}$ in these environments. Calculations were repeated for the zenith and nadir surfaces at achievable, cold end radiator temperatures, specifically $10^{\circ} \mathrm{C}$ and $1^{\circ} \mathrm{C}$ for the conditions specified. 

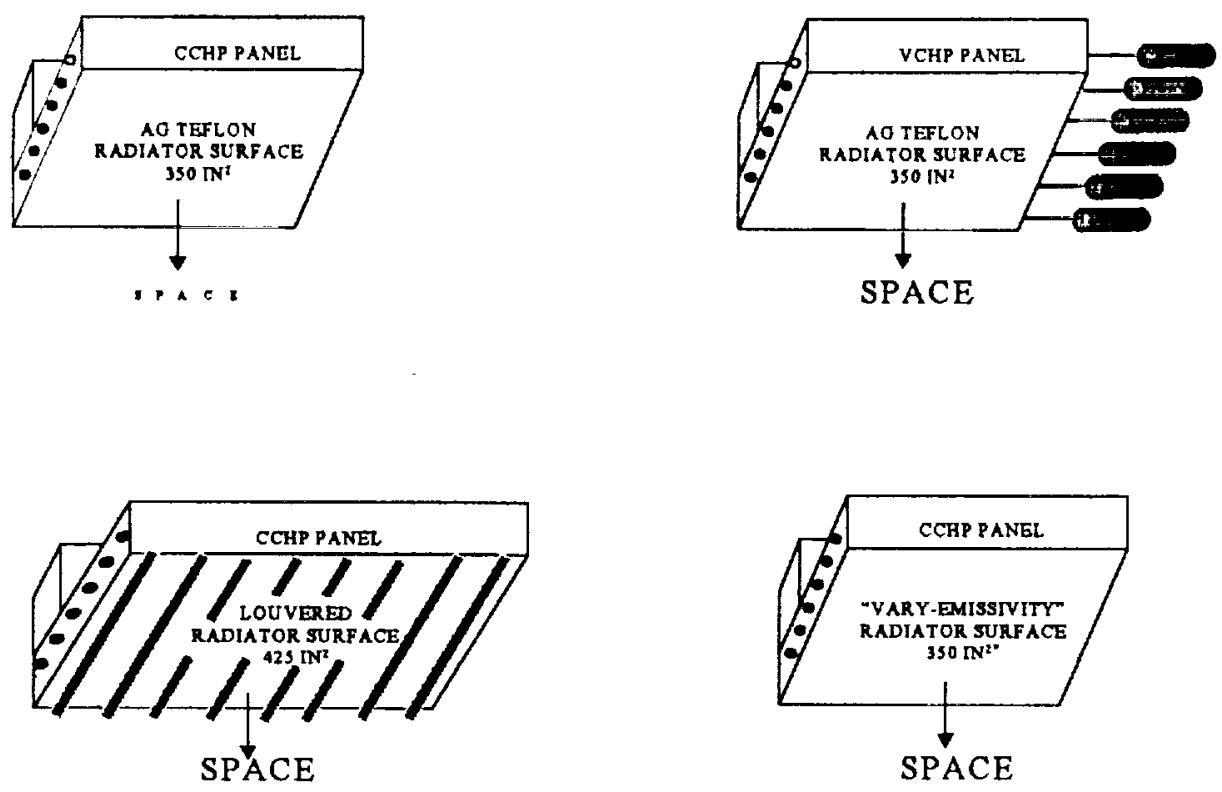

* 350 in 2 and 400 in 2 evaluatod for Eloctrochromic Radiator

Figure 1. Radiator Trade-off Illustrations

\section{Hent Rojoetlon from ZENTH}

32 EETA / BOL AT -10 C

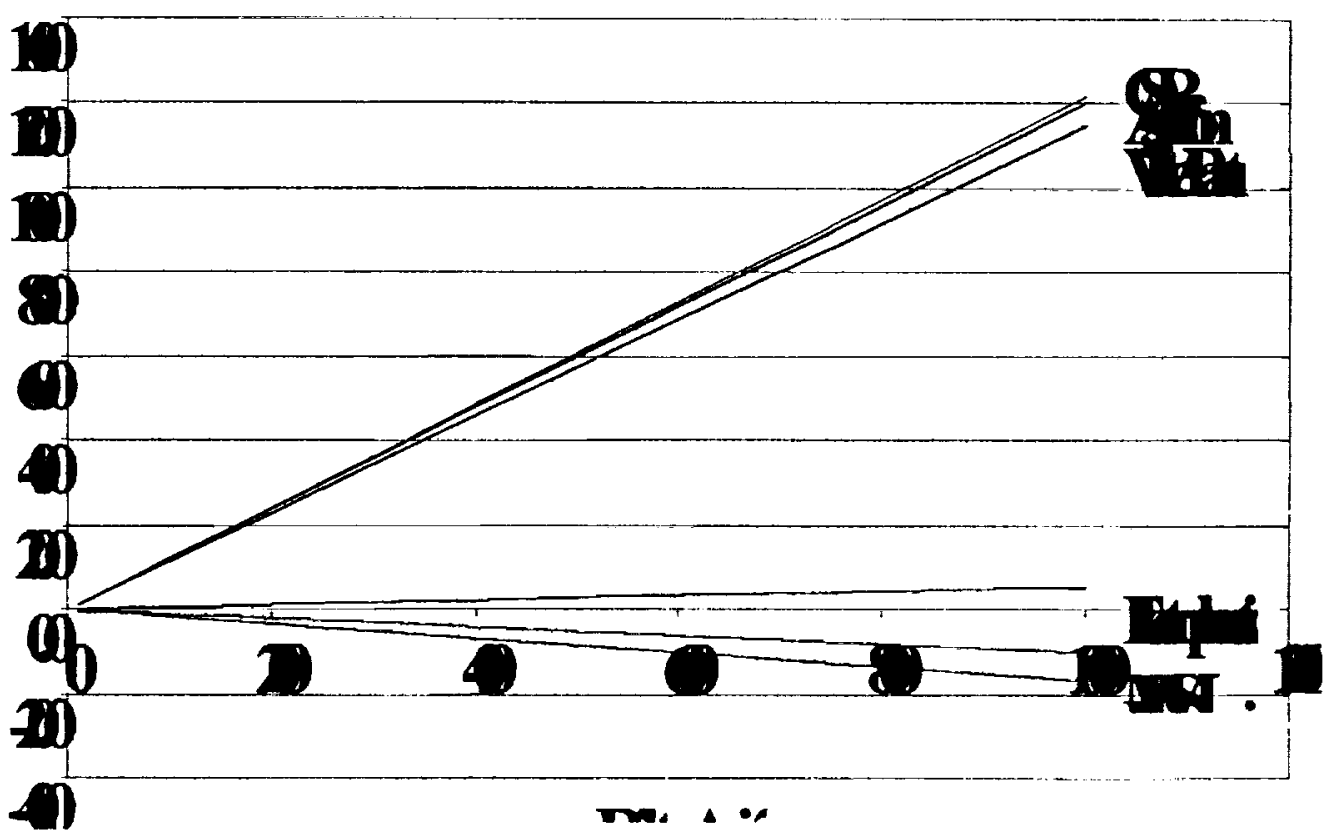

Figure 2. Heat Rejection from Zenith at $-10^{\circ} \mathrm{C} \mathrm{BOL} / 32^{\circ} \mathrm{\beta}$ 
Heet Rejectlon from Zenith

32 BETAIBOLAT $10 \mathrm{C}$

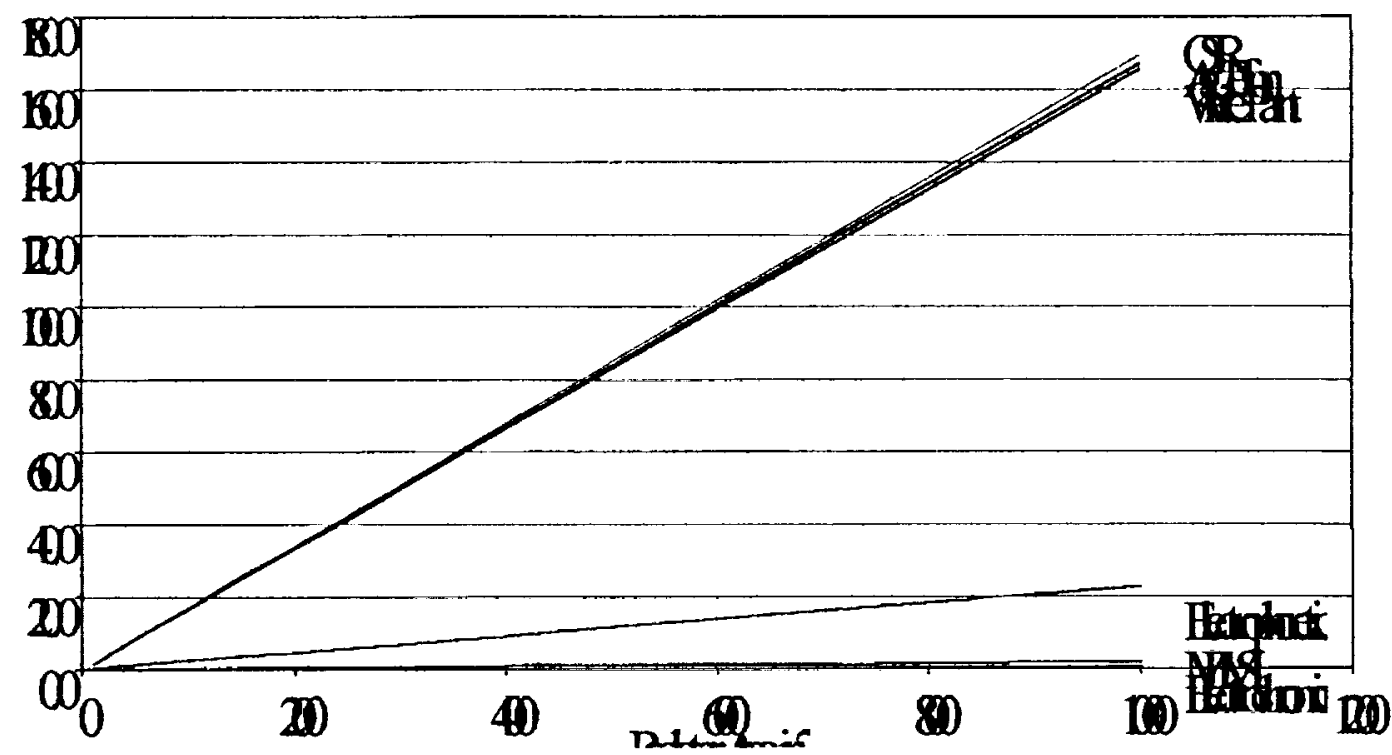

Figure 3. Heat Rejection from Zenith at $10^{\circ} \mathrm{C}$ BOL $/ 32^{\circ} \beta$

Heat Rejection from NADIR

32 BETA BOLAT -10 C

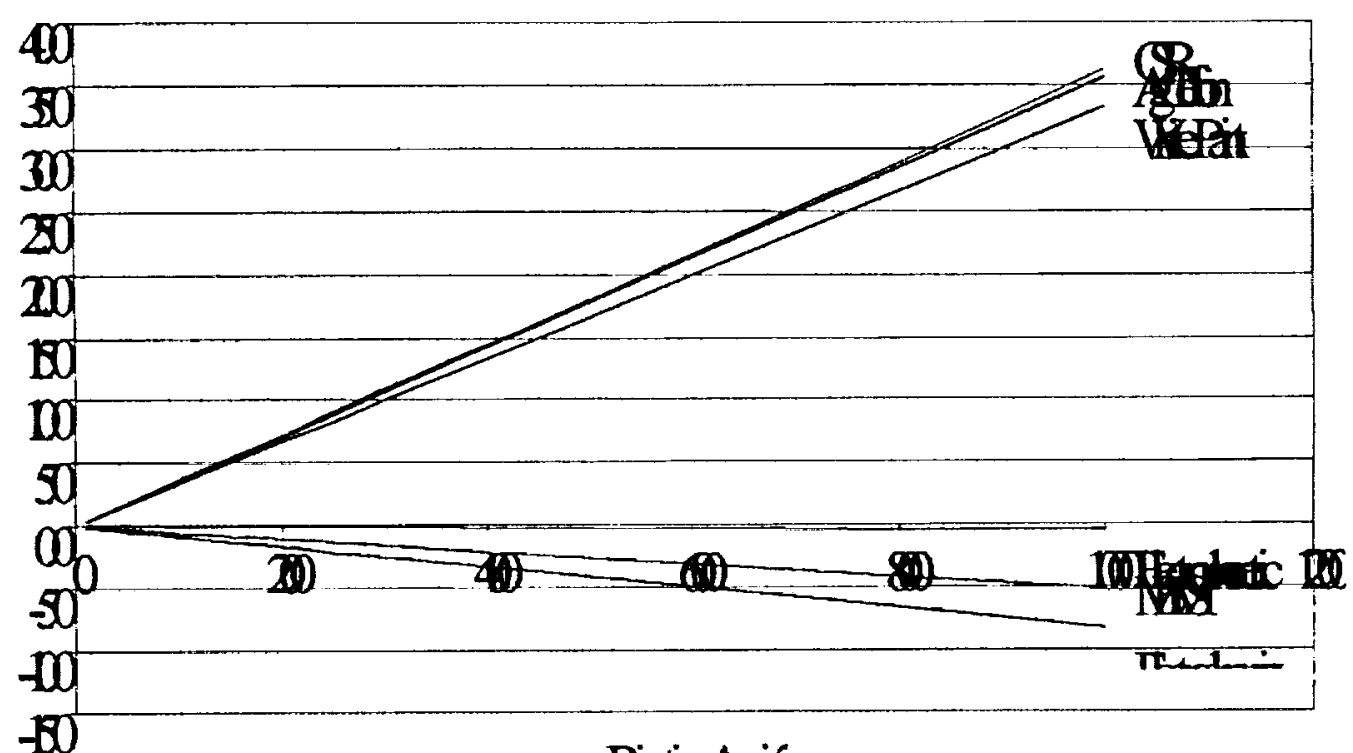

Figure 4. Heat Rejection from Nadir at $-10^{\circ} \mathrm{C} \mathrm{BOL} / 32^{\circ} \beta$ 
The orbital average heater power savings realized for the EOS-AM batteries modeled with the MEMs radiating surface is 54 watts, essentially all the power needed for the batteries to maintain their minimum allowable temperature in the original configuration.

\section{CONCLUSIONS}

As illustrated throughout this study, vary-e technology offers significant advantages over current approaches for radiators in low UV environments. The heater power, mass, and cost savings that can be realized with these systems, is potentially significant for many spacecraft design applications. For example, eliminating the EOS-AM battery heater power during on-orbit hot and cold cases by using a MEMs radiator, demonstrated quite clearly the impact this technology may have on spacecraft design.

In order to make the benefits useful to all satellite radiating surfaces; a reduction in the vary-e's solar absorptivities is needed. Attention must also be paid to the vary-e maximum activation cycles, which may be the limiting factor for battery radiators and other dynamic dissipating systems. A reduction of cycles can be achieved with the proper set point temperature selection and the additional variable switch (i.e. battery pressure, time).

\section{REFERENCES}

J. L. Champion, R. Osiander, M A. Garrison Darrin, T. D. Swanson, "MEMS Louvers for Thermal Control", 1999.

P. Chandrasekhar, "Flexible, Flat Panel Visible/Infrared Electrochromics Based on Conduction Polymers for Space Applications", Ashwin-Ushas Corp., Inc., 1998. 